\title{
Elastic scattering in $n p$ - interactions at intermediate energies
}

\section{Yu.A.Troyan, M.Ch.Anikina, A.V.Belyaev, A.P.Jerusalimov', A.Yu.Troyan}

Joint Institute for Nuclear Research, Dubna, Russia

The study of the elastic np-scattering was carried out at the momenta of incident neutrons $\mathrm{P}_{0}=1.43,1.73$ and $5.20 \mathrm{GeV} / \mathrm{c}$. The obtained results are compared with the available world data. The simple pole model of $\pi$-meson, $\rho$-meson and pomeron exchanges is satisfactory describes the main characteristics of the reaction.

XXI International Baldin Seminar on High Energy Physics Problems JINR, Dubna, Russia

September 10-15, 2012

$1 \quad$ Speaker 


\section{Introduction}

This work continue the study of $n p$-interactions at the momenta of the neutrons $1 \div 5 \mathrm{GeV} / \mathrm{c}$ and is devoted now to the study of elastic scattering.

The world data of elastic np-scattering were obtained many years ago and are very poor and incomplete. It is especially concerned so called charge exchange scattering.

\section{Experiment}

The present study was carried out using the data obtained in an exposure of 1- $m \mathrm{HBC}$ of LHE (JINR) to a quasimonochromatic neutron beam with $\Delta \mathrm{P}_{\mathrm{n}} / \mathrm{P}_{\mathrm{n}} \approx 2.5 \%$ and $\Delta \Omega_{\mathrm{n}} \approx 10^{-7}$ sterad due to the acceleration of deuterons by synchrophasotron of LHE (JINR). The accuracy of the momenta of secondary protons from the reaction is $\Delta \mathrm{P}_{\mathrm{p}} / \mathrm{P}_{\mathrm{p}} \approx 2.0 \%$. The angular accuracy is $\leq 0.5^{\circ}$.

The detailed description of the experiment is published in [1].

The reaction $n p \rightarrow n p$ at the $\mathrm{P}_{\mathrm{n}}=1.43,2.23$ and $5.20 \mathrm{GeV} / \mathrm{c}$ was separated by the standard $\chi^{2}$-method. The selected events were attributed as elastic np-interactions ("El" set) and other inelastic one ("Inel" set). The missing mass distribution has the maximum at the neutron mass and symmetric relatively to its central value.

The separation of elastic events using only missing mass procedure (like in most early studies) leads to an significant admixture of inelastic events $(5 \div 15 \%)$.

Fig. 1 shows the missing mass distribution at $\mathrm{P}_{0}=2.23 \mathrm{GeV} / \mathrm{c}$. Black bins - events from ("Inel" set). The admixture of inelastic events leads to the error in determination of the elastic and inelastic cross-sections and to a distortion of the experimental distributions.

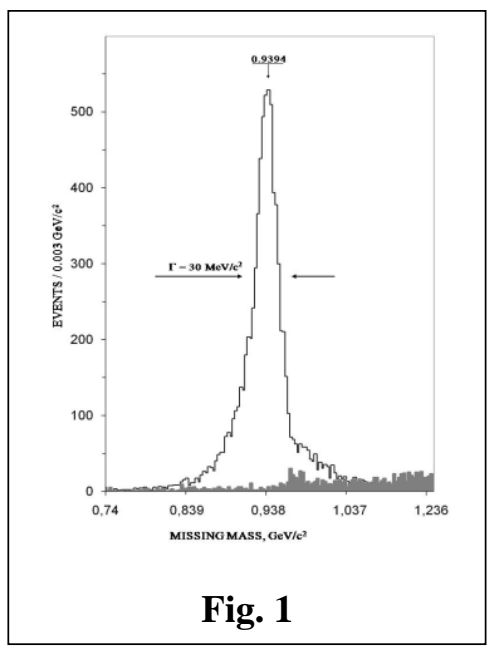

Thus events from "El" set selected by this procedure are characterized by

- $4 \pi$ geometry condition,

- a good determined parameters of primary and secondary particles,

- a negligible admixture of inelastic events. 
Kinematics of the reaction $n p \rightarrow n p$ is shown in Fig. 2

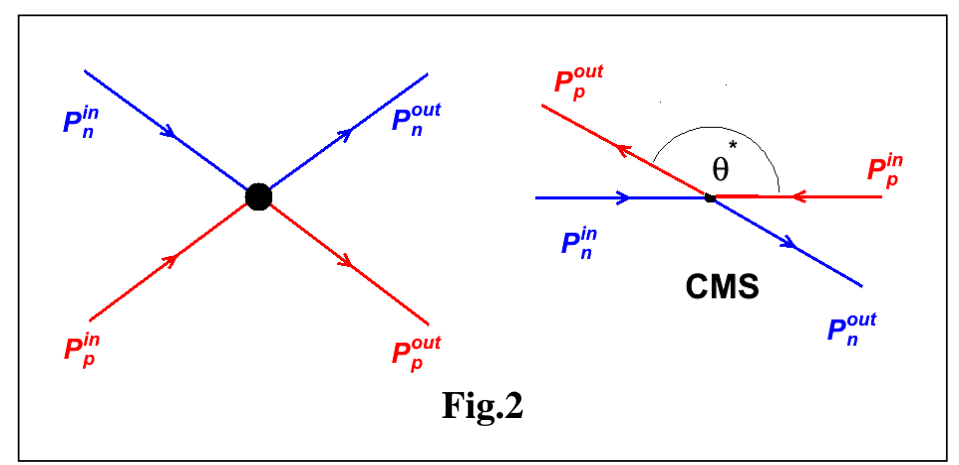

The selected events from "El" set were divided into2 groups:

- Forward scattering $\left(\cos \Theta_{p}{ }^{*}<0\right)$ reaction without charge exchange ("0.ex"). For the further analysis it was taken the variable $\mathrm{t}=\left(\mathrm{P}_{\mathrm{pin}}-\mathrm{P}_{\text {pout }}\right)^{2}$.

- Backward scattering $\left(\cos \Theta_{p}{ }^{*}>0\right)$ reaction with charge exchange ("ch.ex"), main variable $\mathrm{u}=\left(\mathrm{P}_{\text {nin }}-\mathrm{P}_{\text {pout }}\right)^{2}$.

The specifics of the bubble chamber results in the loss of the low energy protons. That leads to a deficit of the events with small 4-momentum transfers $t$ for $\mathrm{P}_{0}=2.23 \mathrm{GeV} / \mathrm{c}$ (Fig.3)

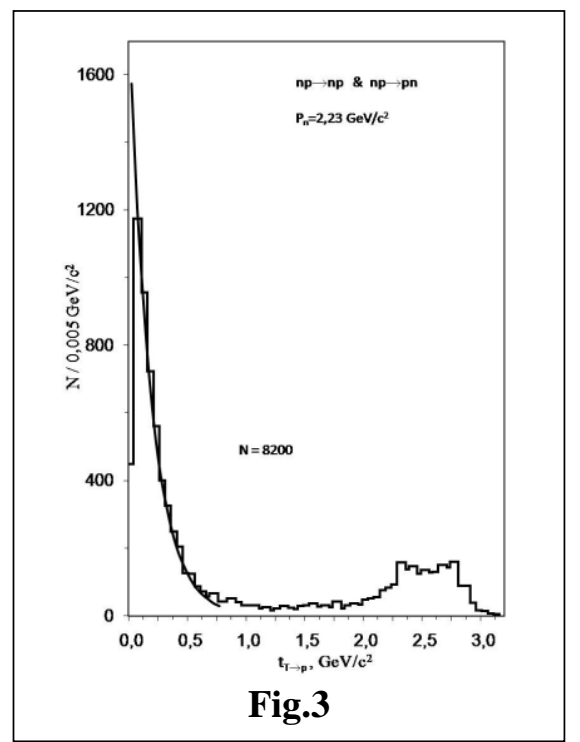

To determine the number of lost events the distribution on $t$ was approximated by the function of the type $\mathrm{Ae}^{-\mathrm{bt}}$ in the range $0.05 \mathrm{GeV}^{2}<|\mathrm{t}|<0.75 \mathrm{GeV}^{2}$.

The extrapolation to the value $|t|=0$ permits to correct the number of elastic events from "El" set.

The determination of the loss for the events from "Inel" set was done by the same manner.

This procedure permits to correct both the number of elastic events and the total number of 1-prong events to calculate the corresponding cross-sections 
The cross-section of the elastic scattering was calculated by the formula

$$
\begin{aligned}
& \sigma_{\mathrm{el}}=\left(N_{e l} / N_{\text {ltot }}\right) \sigma_{1 \text { top }}, \quad \text { where: } \\
& N_{e l}-\text { the number of elastic events, } \\
& N_{\text {ltot }}-\text { the total number of 1-prong events, } \\
& \sigma_{\text {ltop }}-\text { the topological cross-section of 1-prong stars. }
\end{aligned}
$$

The charge exchange cross-sections was calculated by the formula

$\sigma_{\text {ch.ex }}=\left(N_{e l}\left(\cos \Theta_{\mathrm{p}}{ }^{*}>0\right) / N_{e l}\right) \sigma_{\mathrm{el}}$ (no losses of low-energy protons !) - Fig.4b.

The "pure" elastic cross-sections was calculated by the formula

$\sigma_{0 . \mathrm{ex}}=\sigma_{\mathrm{el}}-\sigma_{\text {ch.ex }}-$ Fig. $4 \mathrm{a}$

The results are presented in Fig.4

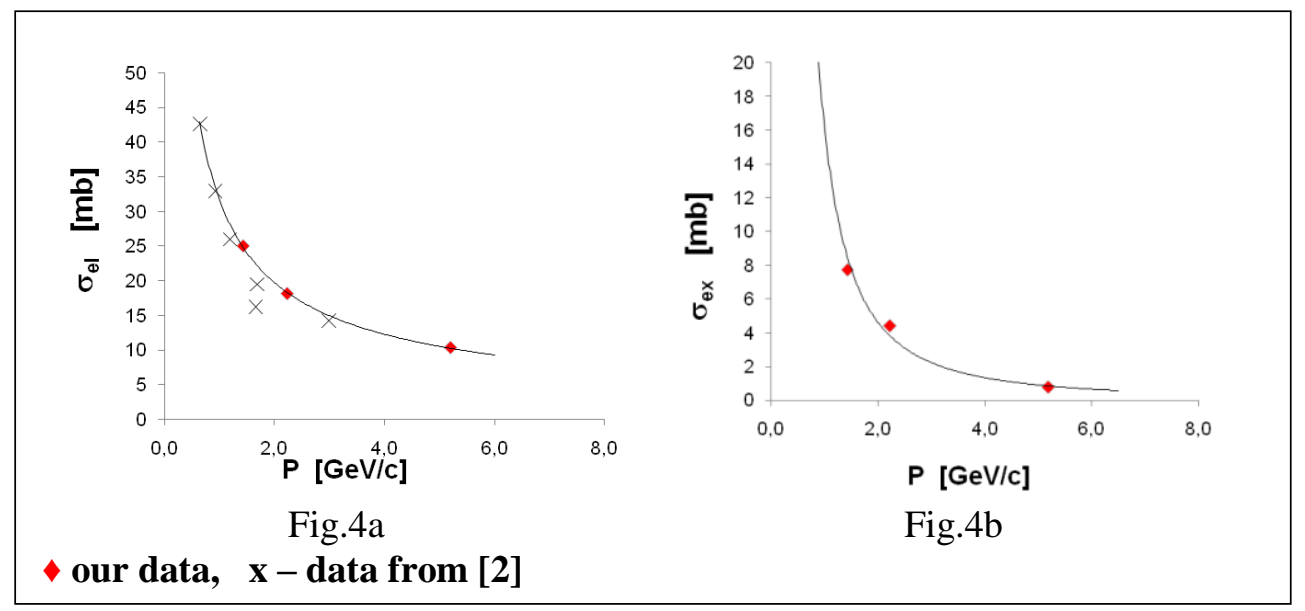

One can see that the data are good approximated by the power functions:

$\sigma_{\mathrm{el}}=31.8 \cdot \mathrm{P}_{\mathrm{n}}^{-0.7}$ and $\sigma_{\text {ch.ex }}=16.3 \cdot \mathrm{P}_{\mathrm{n}}^{-1.8}$.

There were determined the slope parameter b for "pure" elastic ("0.ex") distributions of do/dt (Fig.5):
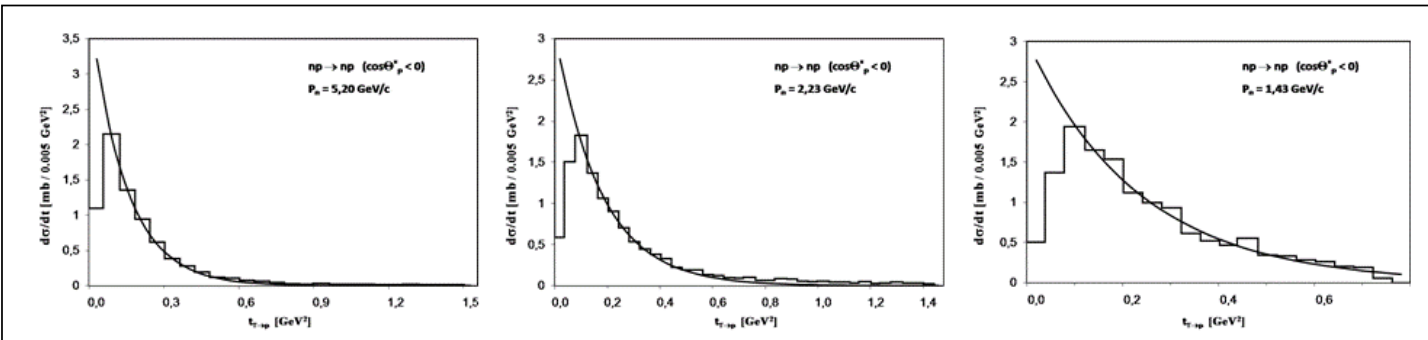

Fig.5 
The slope parameter $\mathbf{b}$ is shown in Fig.6

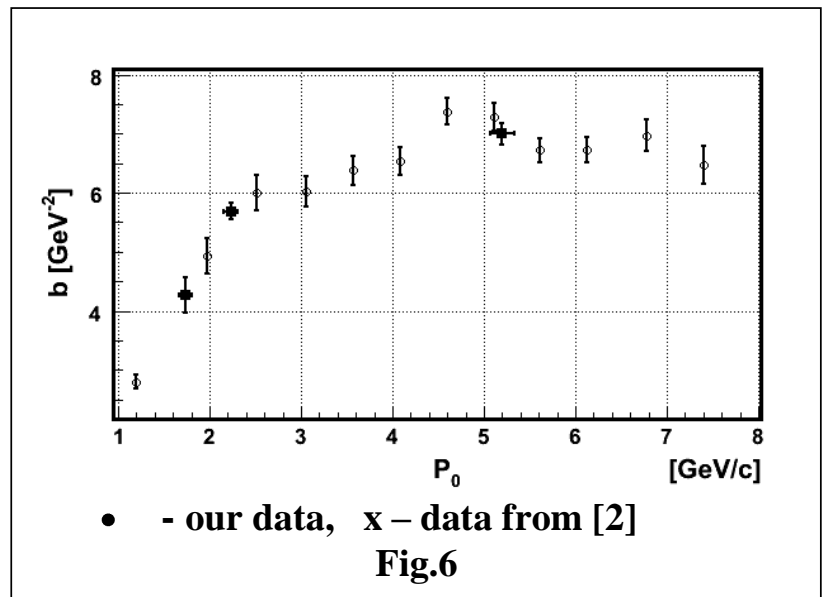

The other results are presented in Table 1

Table 1

\begin{tabular}{|c|c|c|c|}
\hline Pn $[\mathrm{GeV} / \mathrm{c}]$ & 1.43 & 2.23 & 5.20 \\
\hline$\sigma_{\text {el }}[\mathrm{mb}]$ & $25.05 \pm 0.3$ & $18.14 \pm 0.4$ & $10.31 \pm 0.5$ \\
\hline$\sigma_{\text {ch.ex }}[\mathrm{mb}]$ & $7.72 \pm 0,3$ & $4.39 \pm 0.3$ & $0.77 \pm 0.5$ \\
\hline$\sigma_{0 . \mathrm{ex}}[\mathrm{mb}]$ & $17.33 \pm 0.3$ & $13.75 \pm 0.4$ & $9.54 \pm 0.5$ \\
\hline $\mathrm{d}_{0 . \mathrm{ex}} /\left.\mathrm{dt}\right|_{\mathrm{t}=0}\left(\mathrm{mb} / \mathrm{GeV}^{2}\right)$ & 72.6 & 73.3 & 59.3 \\
\hline $\mathrm{d} \sigma_{\text {ch.ex }} /\left.\mathrm{du}\right|_{\mathrm{t}=0}\left(\mathrm{mb} / \mathrm{GeV}^{2}\right)$ & 42.6 & 21.1 & 5.0 \\
\hline
\end{tabular}

\section{Pole model for elastic np scattering}

$\mathrm{np} \rightarrow \mathrm{np}$ reaction is described by the following diagrams (Fig.7a,b).

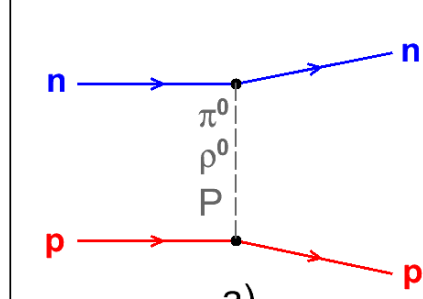

a)

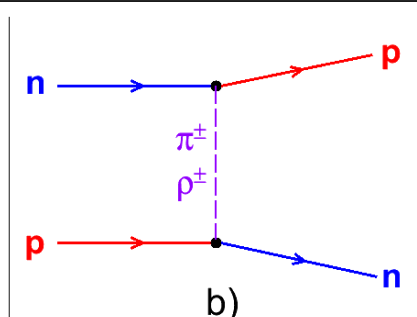

b)

Fig.7

Fig.7a - diagram corresponds to "pure" elastic scattering with exchange by neutral particles: $\pi^{0}$-meson, $\rho^{0}$-meson and pomeron $(\mathrm{P})$.

Fig. $7 \mathrm{~b}$ - diagram corresponds to charge exchange scattering with exchange by charged particles: $\pi^{ \pm}$-meson and $\rho^{ \pm}$-meson.

The exchange by other mesons $\left(\omega^{0}, \mathrm{~A}_{2}\right.$, etc. $)$ does not considered. 
The differential cross-section for the reaction $\mathrm{np} \rightarrow \mathrm{np}$ is written as:

where:

$$
\frac{d \sigma}{d t}=\frac{1}{64 \pi s q^{2}}|T|^{2}
$$

$s$ - square of total energy in CMS,

$q$ - momentum of the incident particles in CMS,

$|T|^{2}$ - square of the matrix element of the reaction.

For charge exchange np scattering the matrix element is written

$$
\left|T_{\text {ch.ex }}\right|^{2}=\left|T_{\pi}+T_{p}\right|^{2}
$$

where:

$$
\begin{aligned}
& T_{\pi}=F \frac{t A_{\pi}}{t-m_{\pi}^{2}} F \text { corresponds to } \pi^{ \pm} \text {-meson exchange; } \\
& T_{\pi}=F \frac{A_{\rho} e^{i \varphi}}{t-m_{\rho}^{2}} F \text { corresponds to } \rho^{ \pm} \text {-meson exchange; }
\end{aligned}
$$

$\mathrm{A}_{\pi}$ and $\mathrm{A}_{\rho}-$ parameters depending only on variable s;

$\varphi$ - shift between $\pi$ and $\rho$ amplitudes of scattering.

Nucleon formfactor $\mathbf{F}$ at each vertex was taken in the form

$$
F(t)=\frac{\Lambda}{\Lambda-t}
$$

and depends only on variable t. Parameter $\Lambda$ was taken equal to $\mathrm{m}^{2}{ }_{\mathrm{N}}$.

In result it was obtained a good description of the differential cross-sections for charge exchange elastic $n p \rightarrow n p$ scattering at 3 energies (Fig.8)
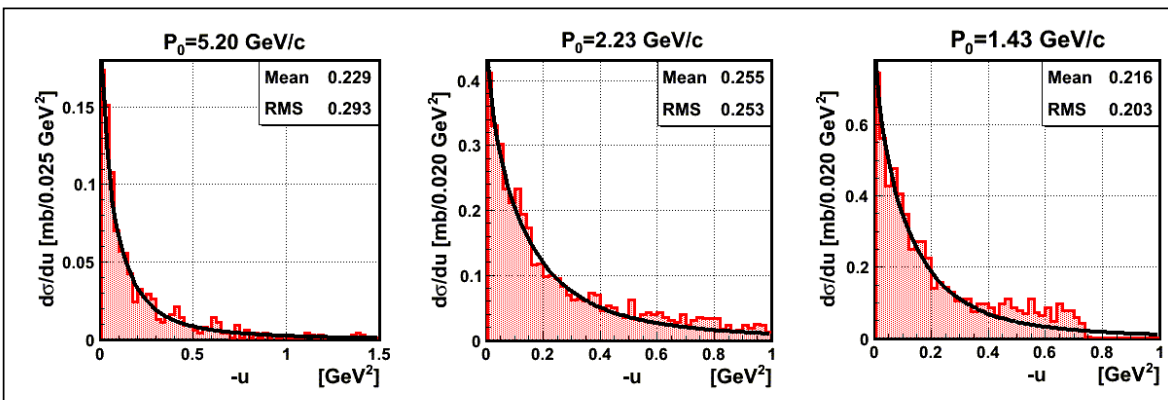

Fig.8

The calculated parameters $\mathbf{A}_{\boldsymbol{\pi}}, \mathbf{A}_{\boldsymbol{\rho}}$ and $\boldsymbol{\varphi}$ (Fig.9) are linearly dependent on ${ }_{\mathbf{s}}$ variable:

$\mathbf{A}_{\boldsymbol{\pi}}=99.3 \mathrm{~V}_{\mathrm{S}-85.7}$ $\mathbf{A}_{\mathbf{p}}=81.7 \sqrt{\mathrm{s}-52.1}$ $\boldsymbol{\varphi}=19.3 \sqrt{ }_{\mathrm{s}}+71.9$
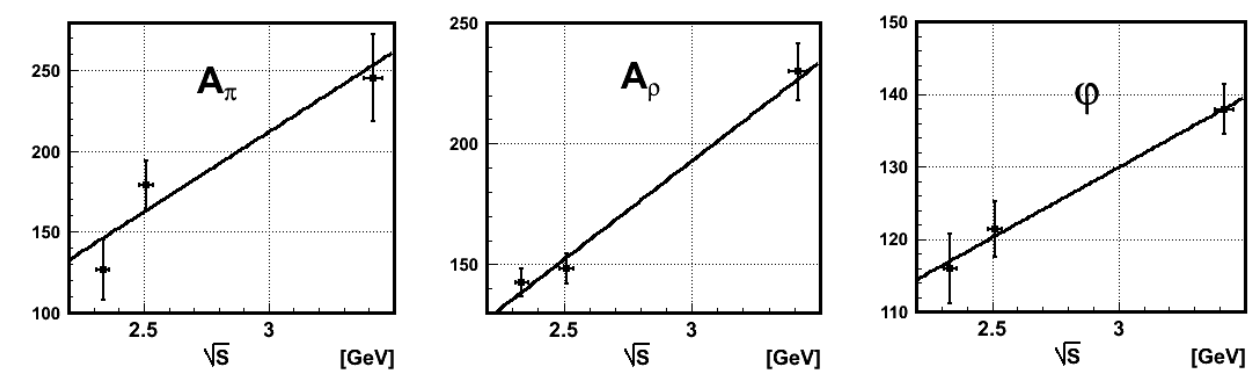

Fig.9 
For "pure" elastic np-scattering the matrix element was chosen in the following form:

$$
\left|T_{{ }^{\prime \prime} e x}\right|^{2}=\frac{1}{4}\left|T_{\pi}+T_{\rho}\right|^{2}+\left|T_{P}\right|^{2},
$$

where $T_{P}=A_{P} e^{b t}$ corresponds to pomeron exchange.

The interference between $(\pi, \rho)$ and P-exchange is negligible.

The factor $1 / 4$ in the expression for $T$ " 0 "ex is due to isotopic relations.

The description of the differential cross-sections for "pure" elastic $n p \rightarrow n p$ scattering is presented in the Fig.10.
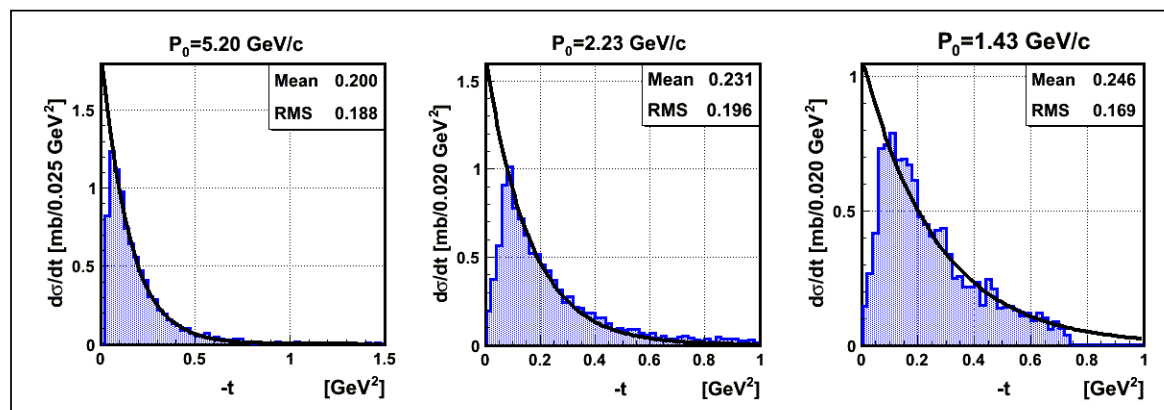

Fig.10

The dependence of the parameters $\mathbf{A}_{\mathbf{P}}$ and $\mathbf{b}_{\mathbf{P}}$ on variable $\mathbf{s}$ are shown in Fig.11.

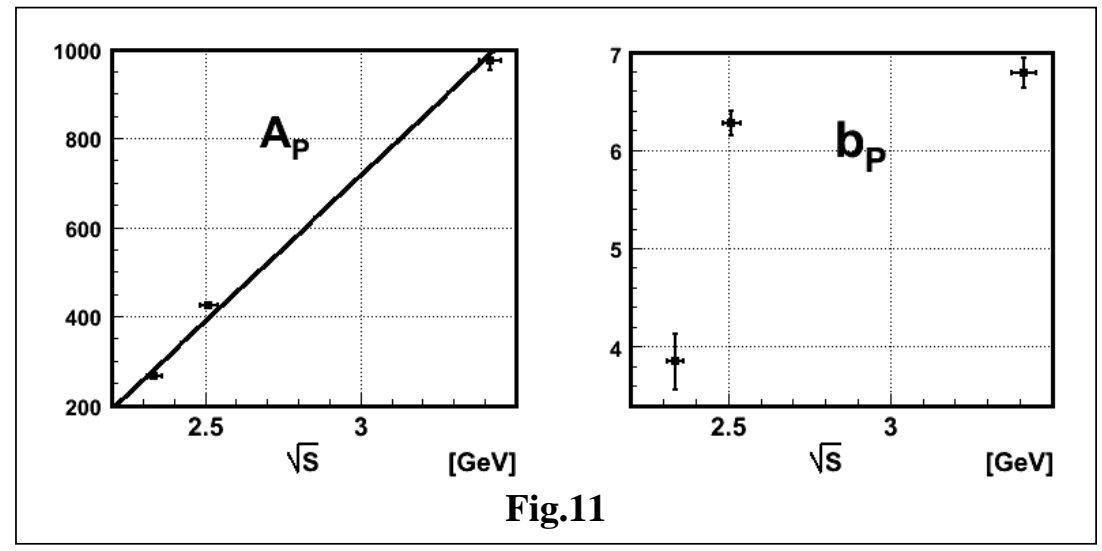

One can see a linearly dependence on variable $\sqrt{S}_{\mathrm{s}}$ for the parameter $\mathbf{A}_{\mathbf{P}}: \mathbf{A}_{\mathbf{P}}=655 \sqrt{\mathrm{S}}_{\mathrm{S}}-1246$

As far as concerned the parameter $\mathbf{b}_{\mathbf{P}}$, its dependence on variable $\mathbf{s}$ is more complicate and may be it needs to construct some special function or to tabulate it.

Thus the presented simple pole model provides a satisfactory description of the characteristics for elastic $n p \rightarrow n p$ scattering at the momenta of incident neutrons $\mathrm{P}_{0}=1.43 \div 5.20 \mathrm{GeV} / \mathrm{c}\left(\mathrm{S}=5 \div 12 \mathrm{GeV}^{2}\right)$.

It was shown that it is necessary take into account the diagrams with pomeron exchange even at relatively small energies.

The model can be used to simulate reaction of elastic $n p \rightarrow n p$ scattering at intermediate energies. 


\section{Conclusion}

- The present work was carried out to study the elastic np-scattering at intermediate energies. The used experimental methods permitted to select elastic events carefully and to do the analysis of them.

- The obtained results essentially supplement the data about the reaction of elastic $n p \rightarrow n p$ scattering especially for charge exchange channel.

- The simple pole model provides a satisfactory description of elastic $n p \rightarrow n p$ scattering and can be used to simulate the reaction at the intermediate energies (for example, for NICA/MPD project [3]).

\section{References}

[1] V. Belonogov et al, A Lliquid Hydrogen Bubble Chamber of Volume $950 \times 350 \times 300 \mathrm{~mm}^{3}$, Nuclear Instruments and Methods, 1963, v. 20 P. 114-115;

A. P. Gasparyan et al., Neutron (Stripping) Channel of JINR of High Energy Laboratory, Prib. Tekh. Eksp., No. 2, 27-42 (1977);

K. Beshliu et al., Channel Cross Sections of np Interaction Reactions with $\mathrm{P}_{\mathrm{n}}=1-5 \mathrm{GeV} / \mathrm{s}$, Yad. Fiz. 63, 888 (1986);

V I. Moroz, A. V Nikitin, and Yu. A. Troyan, Division Methods of Nuclear Reactions Channels under Emission of Bubble Camera by Neutrons with Energy from 2 to $10 \mathrm{GeV}$, Sov. J. Nucl. Phys. 9, 323 (1969);

J. R. Berge, F. T. Solmitz, and H. D. Taft, Kinematical Analysis of Interaction Vertices from Bubble Chamber Data, Rev. Sci. Instrum. 32, 538-548 (1961).

Yu. A. Troyan et al., Seeking and Investigating Isospin Two Narrow Dibaryon Resonances in Neutron-Proton Interactions at $\mathrm{P}_{\mathrm{n}}=5.10 \pm 0.13 \mathrm{GeV} / \mathrm{c}$, Phys. At. Nucl. 63, 1562 (2000)

[2] O. Benary, L. R. Price and G. Alexander, $N N$ and ND Interactions (Above $0.5 \mathrm{GeV} / \mathrm{c}$ ) - $a$ Compilation (PDG). UCRL-20000 NN, August 1970.;

J. Beringer et al,. (Particle Data Group), Phys. Rev. D86, (2012).

[3] http://nica.jinr.ru/. 